\title{
TRANSFER OF GENTAMICIN RESISTANCE BETWEEN CULTURES OF STAPHYLOCOCCUS AUREUS IN NUTRIENT BROTH, SERUM AND URINE
}

\author{
R. W. LACEY AND VICKI L. LORD \\ Department of Pathology, King's Lynn General Hospital, King's Lynn, Norfolk PE30 SOD
}

GENTAMICIN-RESISTANT cultures of Staphylococcus aureus have been reported from many countries since 1975. Although specific epidemics have been associated with bacteria of uniform properties, many distinct strains, as indicated by differences in phage-typing pattern and sensitivity to other antibiotics, have been concerned (Soussy et al., 1975a; Shanson, Kensit and Duke, 1976; Speller et al., 1976; Warren and Roberts, 1976; Bint et al., 1977; Wyatt et al., 1977; Lewis and Altemeier, 1978; Michel, Stessman, and Sacks, 1978; Naidoo and Noble, 1978; Buckwold et al., 1979; Faden et al., 1979; Greenhood et al., 1979).

The sudden appearance of gentamicin resistance in a wide variety of cultures after a 10-year period in which the resistance was exceedingly rare resembles the sequence of events with neomycin resistance in this organism (Lacey, 1971, 1975), and could be explained by the spontaneous transfer of a plasmid or plasmids between cultures in vivo. Gentamicin resistance in $S$. aureus is attributable to the production of inactivating enzymes (Bint et al., 1977; Dowding, 1977; Scott et al., 1978), as is usually the case with plasmid-mediated resistance. Plasmid DNA has been identified in gentamicin-resistant strains by Soussy et al. (1975b) and by Wood, Carter and Best (1977), but in neither case was it established that this DNA was responsible for the resistance, because the plasmid DNA was not isolated after transduction of the trait. Although transduction of gentamicin resistance has been achieved by a few workers, the frequencies have been low (Porthouse et al., 1976; Bint et al., 1977), and several others have been unable to transduce the resistance at all (e.g., Naidoo and Noble, 1978; Greenhood et al., 1979). Naidoo and Noble (1978) showed that gentamicin resistance could be transferred, at rather low frequency, between cultures seeded on to murine or human skin. Recently de Saxe and Porthouse (1979) described the isolation of plasmid DNA after transduction of gentamicin resistance. However, the transduction occurred at rather low frequency and mixed-culture incubation also resulted in transfer of resistance at low frequency.

Thus, as Greenhood et al: (1979) have pointed out, there is strong epidemiological evidence for the occurrence of transfer of gentamicin resistance in nature, but experiments in vitro have not reproduced these postulated events. This paper describes some experiments relevant to this problem.

\section{MATERIALS AND METHODS}

Strains. Twenty-three gentamicin-resistant $S$. aureus strains were obtained from Bristol (4), London (7), Cambridge (5), Birmingham (3) and King's Lynn (4). All were resistant to 
gentamicin (MIC $>5 \mathrm{mg} /$ litre) and tobramycin (MIC $>5 \mathrm{mg} /$ litre). Thrice-purified clones were stored on agar slopes at room temperature without subculture. Other cultures used are described elsewhere (Lacey, 1980); they include the non-lysogenic strain 1030 (Novick, 1967) and 13 derivatives of it, 12 lysogenised by phages of the International Typing Set and one lysogenised by phage $\mathrm{C}$, a transducing phage harboured by culture 13136 .

Nomenclature. The determinant giving resistance to gentamicin and tobramycin is designated gen-r. Prophage carriage is denoted by an arabic number or a letter in parenthesis, chromosomal mutations by unparenthesised capital letters and plasmids in Italic. Thus, culture $1030 \mathrm{SF}(80)$ tet-r contains a chromosomal mutation to streptomycin and fusidic-acid resistance, is lysogenic for phage 80 and carries a plasmid coding for tetracycline resistance. Chromosomal rifampicin resistance is denoted by the letter $R$.

Mixed-culture transfer, mitomycin-C induction, transduction, phage propagation, filtration of lysates, antibiotic-sensitivity testing and bacteriophage typing were performed as described by Lacey (1980). When gentamicin resistance was transferred successively through clones of strain 1030, at least two subcultures were made on antibiotic-free agar between each transfer. Gentamicin-resistant colonies were selected, after incubation in mixed culture on nutrient agar containing gentamicin $2.5 \mathrm{mg} /$ litre and either fusidic acid $10 \mathrm{mg} /$ litre and streptomycin 100 $\mathrm{mg} /$ litre, or rifampicin $100 \mathrm{mg} /$ litre, as appropriate. Transductants from cell-free lysates were isolated on nutrient agar containing gentamicin $2.5 \mathrm{mg} /$ litre. The frequency of transfer in mixed culture was expressed as the number of resistant recipients/the final number of donors, and transduction frequency as the number of resistant recipients/the number of plaque-forming units (p.f.u.) in the phage lysate.

Serum and urine. Pooled human serum (Dade No. XLT 373, Didcot, Oxfordshire) was used. Urine was obtained from patients with untreated urinary-tract infections, then pooled, filtered, and checked for sterility. Pools consisted of from five to seven 10-ml urine samples from different patients.

Replica plating was performed on nutrient agar containing gentamicin $2.5 \mathrm{mg} /$ litre.

\section{RESULTS}

\section{Screening for transferable gentamicin resistance}

The 23 gentamicin-resistant cultures were each incubated in mixed culture with rifampicin-resistant variants of strain 1030 and each of its 13 lysogenic derivatives. No transfer of gentamicin resistance occurred from 22 of the cultures, but one culture (no. G18) transferred its resistance to each of the recipients at frequencies of $10^{-3}-10^{-4}$ (table I). Culture G18 had originally been isolated in Birmingham (Bint et al., 1977), and is resistant to gentamicin (MIC: $10 \mathrm{mg} /$ litre) and to tobramycin (MIC: $10 \mathrm{mg} /$ litre) but not to amikacin (MIC: $2 \mathrm{mg}$ /litre). Resistance is due to adenylation and to acetylation of gentamicin and tobramycin (Bint et al., 1977).

\section{Properties of gentamicin-resistant recipients}

Three clones of each of the recipients that had acquired gentamicin resistance in mixed culture were initially studied. All were as resistant to concentrations of gentamicin and tobramycin, as the donor culture G18, but there was no alteration in sensitivity to other antibiotics including neomycin, streptomycin and amikacin. The phage-typing pattern of each resistant recipient was unaltered except that all of the lysogenic derivatives of strain 1030 that were 
TABLE I

Frequency of transfer of gentamicin resistance in mixed culture from the wild strain G18 to the non-lysogenic strain 1030R and to lysogenised cultures of it

\begin{tabular}{l|c}
\hline $\begin{array}{c}\text { Recipient } \\
\text { culture no. }\end{array}$ & $\begin{array}{c}\text { Frequency of transfer } \begin{array}{c}\text { to the } \\
\text { indicated recipient culture }\end{array} \\
1030 \mathrm{R}\end{array}$ \\
$1030 \mathrm{R}(\mathrm{C})$ & $8.5 \times 10^{-4}$ \\
$1030 \mathrm{R}(52)$ & $1.1 \times 10^{-4}$ \\
$1030 \mathrm{R}(79)$ & $5.2 \times 10^{-4}$ \\
$1030 \mathrm{R}(80)$ & $1.2 \times 10^{-4}$ \\
$1030 \mathrm{R}(55)$ & $7.0 \times 10^{-4}$ \\
$1030 \mathrm{R}(71)$ & $9.4 \times 10^{-4}$ \\
$1030 \mathrm{R}(95)$ & $4.7 \times 10^{-4}$ \\
$1030 \mathrm{R}(42 \mathrm{E})$ & $9.4 \times 10^{-4}$ \\
$1030 \mathrm{R}(54)$ & $8.0 \times 10^{-4}$ \\
$1030 \mathrm{R}(77)$ & $7.2 \times 10^{-4}$ \\
$1030 \mathrm{R}(81)$ & $7.6 \times 10^{-4}$ \\
$1030 \mathrm{R}(88)$ & $1.2 \times 10^{-5}$ \\
1030R(96) & $3.7 \times 10^{-4}$ \\
& $2.9 \times 10^{-4}$ \\
\hline
\end{tabular}

* $\mathrm{R}=$ chromosomal resistance to rifampicin; in parentheses, lysogenising phage.

$\dagger$ Number of resistant receptors/final number of donors.

lysed by typing phage 75 became resistant to this phage on acquiring gentamicin resistance. One lysogenic derivative of strain 1030-culture 1030(80)-is non-inducible by mitomycin C 2.0-10 mg/litre (Lacey, 1980). However, each of the three clones of this culture became readily inducible with these concentrations of mitomycin $\mathrm{C}$ on gain of gentamicin resistance. Non-lysogenic wild strain 1030 did not become inducible by mitomycin C $2 \cdot 0,5 \cdot 0$, or $10 \mathrm{mg} /$ litre when it acquired gentamicin resistance.

Transfer of gentamicin resistance between clones of the non-lysogenic strain 1030

Ten clones of culture 1030R gen-r obtained after mixed culture with strain G18 (table I) were incubated with culture 1030SF. In each mixture, gentamicin resistance was transferred at a frequency of $\sim 10^{-4}$. After thrice plating on nutrient agar, the resistance was then transferred from 20 clones of culture $1030 \mathrm{SF}$ gen-r to culture 1030R at similar frequency. Twenty clones of the newly constructed culture $1030 \mathrm{R}$ gen- $r$ were then mixed with culture 1030SF; transfer of resistance occurred at frequencies as above from 19 clones, but one clone of 1030R gen-r failed to transfer its resistance. This clone, designated 1030R gen-r X, will be described in detail below.

It thus appeared that gentamicin resistance could transfer spontaneously between non-lysogenic derivatives of strain 1030. Under these conditions, the chromosomal genes $\mathrm{F}, \mathrm{S}$ and $\mathrm{R}$ do not transfer spontaneously between nonlysogenic clones of 1030 (Lacey, 1980); the direction of transfer is therefore 
assumed to be from the putative gentamicin-resistant donor to the recipient. The final 19 resistant recipients (1030SF gen-r) were examined in detail. All were resistant to typing phage 75 . Three cultures of 1030SF gen- $r$ were studied to determine whether phage 75 became adsorbed to them. As with the wild strain 1030 , all cultures rapidly adsorbed this phage (and also phage 55) so that, after incubation of $\sim 10^{9}$ cells in $0.01 \mathrm{M} \mathrm{CaCl}_{2}$ with about $10^{6}$ p.f.u. of the phages for $30 \mathrm{~min}$. at $37^{\circ} \mathrm{C},>95 \%$ of phage was lost on filtering. Controls included cultures containing $0.02 \mathrm{M}$ sodium citrate. Resistance to lysis by phage 75 is therefore probably due to lysogenic immunity and not to failure of phage adsorption. The 19 cultures $1030 \mathrm{SF}$ gen-r each contained a low titre of phage particles in culture supernatants $\left(\sim 10^{1}-10^{3}\right.$ p.f.u. $\left./ \mathrm{ml}\right)$. Although non-inducible by mitomycin C $2 \cdot 0-10 \cdot 0 \mathrm{mg} /$ litre, they showed partial clearing after treatment with lower concentrations $(0 \cdot 2-0 \cdot 5 \mathrm{mg} /$ litre $)$. Filtrates of these lysates contained $\left(6 \times 10^{4}\right)-10^{8}$ p.f.u. of phage $/ \mathrm{ml}$ (titred on 1030 wild).

Thus, the cultures 1030SF gen-r were lysogenic as shown by (1) resistance to lysis by phage $75,(2)$ inducibility by low concentrations of mitomycin $\mathrm{C}$ and (3) the presence of phage particles in culture filtrates. Because these properties were almost always associated with gentamicin resistance, it is likely that genes coding for this resistance were covalently linked to prophage genome. Transfer in mixed culture required either $0.01 \mathrm{M}$ calcium or magnesium ions and was abolished by $0.02 \mathrm{M}$ trisodium citrate. The properties of gentamicin-resistant derivatives obtained by mixed culture were therefore similar to those obtained by transduction by a cell-free lysate (see below).

\section{Transfer of gentamicin resistance in serum and urine}

Cultures 1030R gen-r and 1030SF were incubated together in nutrient broth, pooled serum and pooled urine. Gentamicin-resistant derivatives of 1030SF were identified. The transfer frequency observed in urine $\left(10^{-3}-10^{-4}\right)$ was similar to that in nutrient broth. However, a much higher frequency was observed in serum $\left(\sim 10^{-1}-10^{0}\right)$ although the final numbers of donors and recipients were reduced. Typical results are shown in table II. The addition of increasing amounts of serum to nutrient broth had little effect on the

TABLE II

Transfer of gentamicin resistance (gen-r) between clones of strain 1030 in serum*

\begin{tabular}{|c|c|c|c|}
\hline \multirow{2}{*}{ Experiment no. } & \multicolumn{3}{|c|}{$\begin{array}{l}\text { Number of colony-forming units } \\
\text { (c.f.u.)/ml at } 24 \mathrm{~h} \text { of }\end{array}$} \\
\hline & $\begin{array}{c}\text { donor } \\
\text { (1030Rgen-r) }\end{array}$ & $\begin{array}{l}\text { sensitive } \\
\text { recipient } \\
(1030 \mathrm{SF})\end{array}$ & $\begin{array}{l}\text { resistant } \\
\text { recipient } \\
(1030 \text { SF gen-r) }\end{array}$ \\
\hline $\begin{array}{l}1 \\
2\end{array}$ & $\begin{array}{l}2.0 \times 10^{4} \\
3.2 \times 10^{4}\end{array}$ & $\begin{array}{l}2.4 \times 10^{5} \\
5.2 \times 10^{5}\end{array}$ & $\begin{array}{l}5.4 \times 10^{4} \\
7.2 \times 10^{4}\end{array}$ \\
\hline
\end{tabular}

$\mathbf{R}, \mathbf{S}$, and $\mathbf{F}=$ chromosomal resistance respectively to rifampicin, streptomycin and fusidic acid.

* Initial concentration of donor and recipient $10^{7} \mathrm{c.f} . \mathrm{u} . / \mathrm{ml}$. 
frequency of transfer until about $50 \%(\mathrm{v} / \mathrm{v})$ of serum was added (data not shown).

\section{Transduction of gentamicin resistance}

Donor cultures G18, 1030SF gen-r and 1030R gen-r were induced with mitomycin $\mathrm{C}$; the lysates were checked for sterility and used to transduce gentamicin resistance to culture 1030SF. Each unirradiated lysate gave a transduction frequency of about $10^{-5}$. The effect of irradiation with ultraviolet light was to increase this frequency to a maximum of between $4 \cdot 2$-fold for culture G18 and $6 \cdot 0$-fold for culture 1030R gen-r, with a fall in phage titre by about one log. Frequency for transduction of gen-r from culture $1030 \mathrm{R}$ gen-r to culture 1030SF and various lysogenic derivatives is shown in table III. As with mixed-culture transfer, lysogeny had no effect on the rate of transduction.

Gentamicin resistance was transduced from an unirradiated mitomycin-Cinduced lysate of donor culture 1030SF gen-r to culture 1030SF. Twenty transductants were examined in detail (table IV). Because lysogeny had been found to be associated with increased sensitivity to sulphonamides and trimethoprim (Lacey, 1980), the 1030SF gen- $r$ derivatives were tested for sensitivity to these agents. Eleven had become more sensitive to both sulphadiazine and trimethoprim than culture 1030SF (table IV). All possessed the phenotype 1030SF gen-r, were also resistant to tobramycin but sensitive to amikacin, and were resistant to lysis by phage 75 . Nineteen contained plaque-forming activity in their culture filtrates, were inducible by low concentrations of mitomycin $\mathrm{C}$ and could transfer their gentamicin resistance, at the typical frequency of $10^{-3}-10^{-4}$, to culture $1030 \mathrm{R}$. However, one culture (1030SF

TABLE III

Frequency of transduction of gentamicin resistance from mitomycin-C-induced lysate of culture 1030SF gen-r to culture 1030R and to lysogenic derivatives of it*

\begin{tabular}{l|c}
\hline Recipient culture no.* & $\begin{array}{c}\text { Frequency of transduction to } \\
\text { the indicated recipient culture }\end{array}$ \\
\hline 1030R & $3.3 \times 10^{-6}$ \\
1030R(C) & $2.6 \times 10^{-6}$ \\
1030R(52) & $5.8 \times 10^{-6}$ \\
1030R(79) & $8.5 \times 10^{-6}$ \\
1030R(80) & $4.5 \times 10^{-6}$ \\
1030R(55) & $3.6 \times 10^{-6}$ \\
1030R(71) & $9.1 \times 10^{-6}$ \\
1030R(95) & $1.4 \times 10^{-5}$ \\
1030R(42E) & $8.2 \times 10^{-6}$ \\
1030R(54) & $4.5 \times 10^{-6}$ \\
1030R(77) & $2.3 \times 10^{-6}$ \\
1030R(81) & $2.3 \times 10^{-6}$ \\
1030R(88) & $2.2 \times 10^{-6}$ \\
1030R(96) & $7.2 \times 10^{-6}$ \\
\hline
\end{tabular}

* For designation of cultures see tables I and II.

$\dagger$ Number of resistant recipients/number of plaque-forming units in the lysate. 
gen-r-20; see table IV), subsequently referred to as $1030 \mathrm{SF}$ gen-r $\mathrm{Z}$, possessed no plaque-forming activity in its culture supernatant, was non-inducible with mitomycin $C$ and could not transfer its gentamicin resistance to culture 1030R. However, the gen- $\mathrm{Z}$ clone showed increased sensitivity to sulphadiazine and trimethoprim, and was resistant to lysis by phage 75. Thus, some of the phage function of the element gen-r had been lost.

Clones 1030SF gen-r-1, gen-r-2, and gen-r-4 (MIC of sulphadiazine 40.0, $7 \cdot 5,30.0 \mathrm{mg} /$ litre respectively) were induced with mitomycin $\mathrm{C}$ and the sterile lysates were used to transduce gen-r to $1030 \mathrm{R}$. Each series of resultant transductants showed variable sensitivity to sulphadiazine (MIC either 5.0-7.5 $\mathrm{mg} /$ litre or $30-40 \mathrm{mg} / \mathrm{litre}$ ) that was not correlated with the sensitivity of the donor clones.

The clone 1030R gen-r $\mathrm{X}$ that had been obtained during mixed-culture incubation (see above) had properties similar to those of the clone 1030SR gen-r $\mathrm{Z}$. Transducing phage $\mathrm{C}$ was propagated on both of these and used to transduce gentamicin resistance to culture 1030SF. As above, kinetics were typical for a chromosomal gene; ultraviolet irradiation stimulated a rather low initial frequency $\left(\sim 10^{-6}\right)$ about fivefold.

\section{Stability of gentamicin resistance on storage}

Cultures 1030R gen-r, 1030SF gen-r and 1030(80) gen-r were incubated for 4 weeks at $37^{\circ} \mathrm{C}$ statically in nutrient broth. Portions of each were then subcultured and incubated similarly for $24 \mathrm{~h}$ at $37^{\circ} \mathrm{C}$, diluted and examined for loss of resistance by replica plating. No resistance-loss was seen from about 2000 colonies of each culture examined.

Can the gentamicin-resistance determinant mediate transfer of other resistances?

Plasmids coding for either penicillinase production (pen-r) or tetracycline resistance (tet-r) were transduced separately from a mitomycin-C induced culture of strain 13136 to 1030R gen-r. Six of each pen-r and tet-r derivative were incubated with culture 1030SF in nutrient broth and in pooled serum. Whilst gentamicin resistance transferred from each clone at the expected frequencies, neither pen-r or tet-r could be transferred. It had previously been found that under these conditions pen-r or tet-r could be transferred from strain 13136 to strain 1030 but not between non-lysogenic clones of strain 1030 (Lacey, 1980).

\section{Can gen-r inhibit plasmid transfer?}

Because gen-r contains phage genome, its presence in the donor cell might inhibit transfer of plasmids (Lacey, 1980). To test for this, gen- $r$ was transduced from culture 1030R gen-r into cultures $1030 \mathrm{cp}-2$ and $1030 \mathrm{cp}-3$, which contain presumptive plasmids able to mediate their own transfer at very high frequency (Lacey, 1980). The presence of gen-r was found to reduce the 


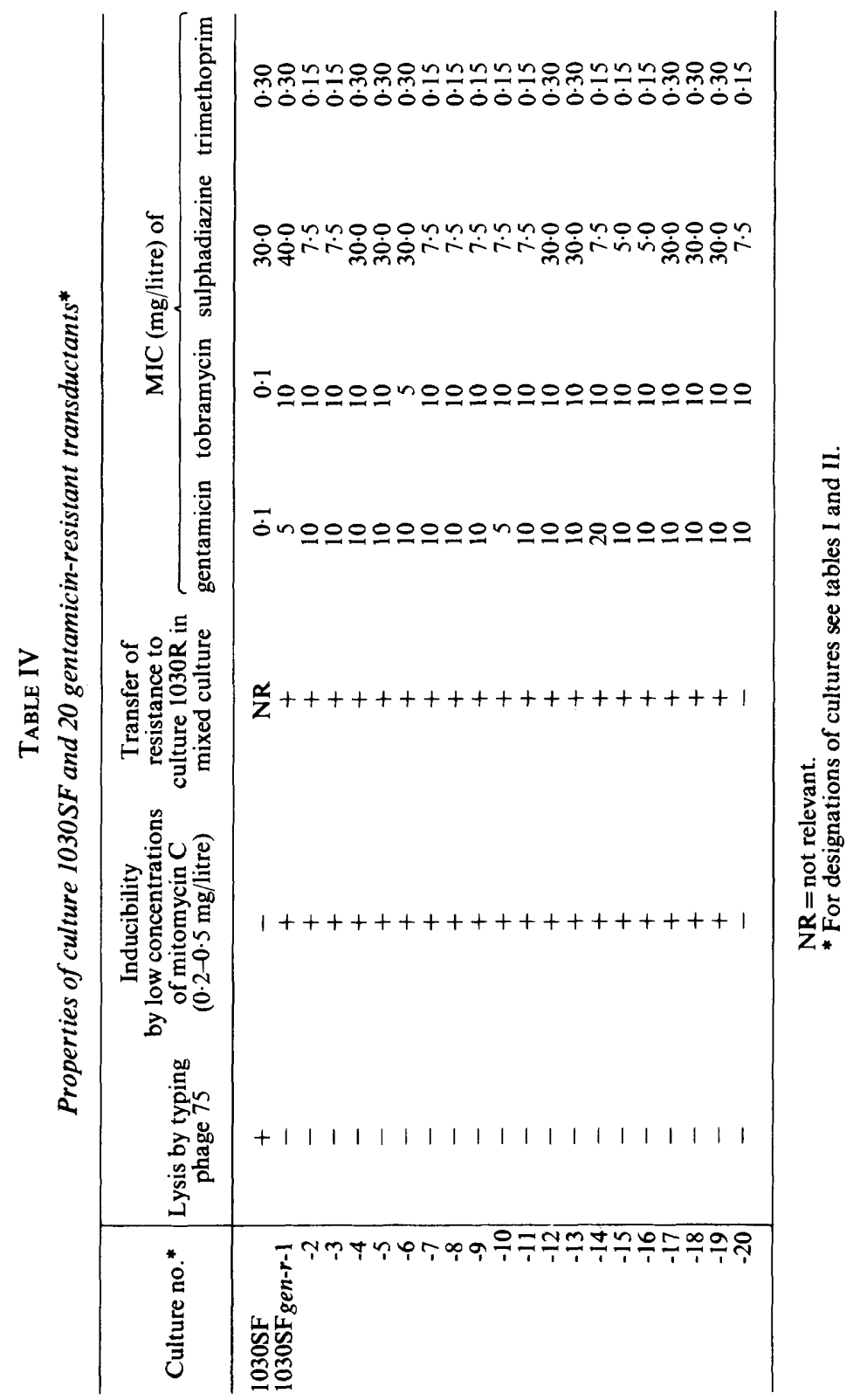


transfer-frequency in nutrient broth of $c p-2$ and $c p-3$ to culture $1030 \mathrm{~F}$ from about $10^{-1}$ for cultures $1030 c p-2$ and $1030 c p-3$ to $10^{-3}$ for cultures $1030 c p-2$ gen-r and $1030 \mathrm{cp}-3$ gen-r.

\section{Discussion}

Only one of 23 naturally occurring gentamicin-resistant cultures of $S$. aureus examined has been found to transfer its resistance spontaneously to derivatives of strain 1030 . Thereafter this resistance passed spontaneously between clones of strain 1030 at frequencies of $c \cdot 10^{-3}-10^{-4}$. Because the wild strain 1030 is non-lysogenic and bacteriophages appear to be necessarily concerned in gene transfer in this culture (Lacey, 1975, 1980), the spontaneous transfer of an element through several clones of a non-lysogenic culture implies that the element can in some way mediate its own transfer. The element gen-r does indeed harbour phage genome, as shown by the inducibility of cultures harbouring it, the presence of plaque-forming units in their filtrates and resistance to typing phage 75 . The mechanism of this transfer is not known with certainty. Because the frequencies of spontaneous transfer in mixed culture and of transduction are not affected by lysogeny in the recipients, the actual transfer vector may be bacteriophages themselves. However, it has not been possible to find particles capable of transferring gentamicin resistance from uninduced culture filtrates. This may be a matter of frequency, because the transduction frequency from lysates of induced cultures is typically about $10^{-5}$. Alternatively, the transferring particles may be cell bound or unstable (Lacey, 1975). Bacteriophages can also be concerned in a different form of gene transfer, described as conjugation (Lacey, 1980) whereby a prophage in either the donor or recipient can-presumably by causing cell-to-cell adhesion-permit plasmids to be transferred between cells. The prophage component of gen-r cannot effect spontaneous transfer of other plasmids within the cell to a recipient. This, together with the finding that lysogeny in the recipient has little effect on transfer, suggests that spontaneous transduction of gen-r rather than conjugation is the operative mechanism.

It is not possible to conclude from the above data whether gen-r is mediated by a conventional plasmid or a pseudoplasmid (Phillips and Novick, 1979), or is chromosomal. The stability of the resistance and transduction kinetics favour a chromosomal locus; the presence of a prophage component is also consistent with this. One curious finding was that the presence of the element gen-r was not always associated with increased sensitivity to sulphadiazine and trimethoprim, whereas lysogeny with 13 other phages was always associated with increased sensitivity to these two agents (Lacey, 1980). This suggests that gen-r may become integrated into the chromosome at different loci, only one of which causes increased sulphonamide sensitivity. Transduction into a rec ${ }^{-}$ cell will give little information because plasmids and chromosomal transposons can be established there (Phillips and Novick, 1979).

From the practical point of view, the isolation of an element coding for gentamicin resistance that can apparently transfer itself through several clones 
of $S$. aureus may be of importance in relation to antimicrobial therapy. Because the transfer occurs in urine at frequencies of $\sim 10^{-4}$ and in serum at frequencies of $\sim 10^{-1}$, transfer might well occur in vivo, notably on the body surface. Such transfer has been demonstrated experimentally by Naidoo and Noble (1978) and the use of topical gentamicin would be expected to select resistant recipients in vivo. In view of the large number of other topical antibiotics, and of disinfectants, that are available, the argument for careful restriction in the use of topical gentamicin seems powerful. The high frequency of transfer in serum is not readily explained. However, the total numbers of bacteria after incubation for $24 \mathrm{~h}$ were low and it is possible that the antibacterial effects of serum might also cause induction of the donor with release of transducing phages.

Two clones of strain 1030 gen-r were identified, one after mixed-culture transfer and one after transduction, that were unable to transfer the resistance further in mixed culture. Some, but not all, of the prophage function had been lost. The genes determining gentamicin resistance in these two cultures are probably chromosomal. The difficulty experienced by several groups of workers in transducing gentamicin resistance might be explained if gen-r passed spontaneously between cells in vivo but its phage component tended to be lost in culture. On entry into the recipient cell, the genes determining gentamicin resistance would survive only if they became integrated into the chromosome or into a plasmid - as presumably had occurred in the cultures described by de Saxe and Porthouse (1979) and possibly others (Soussy et al., $1975 b$; Wood, Carter, and Best, 1977). If such loci have a low probability of inclusion into prophages during induction or propagation, transduction would be difficult. These postulated events imply that the genes determining gentamicin resistance comprise a transposon. Phillips and Novick (1979) have recently described a transposon in $S$. aureus that determines resistance to erythromycin and spectinomycin, and has prophage functions, that becomes integrated into the chromosome. Futher studies are required to establish whether gentamicin resistance can also be considered transposable.

\section{SUMMARY}

Only one of 23 gentamicin-resistant cultures of Staphylococcus aureus transferred its resistance in mixed culture in broth to the non-lysogenic $S$. aureus strain 1030; the transfer-frequency was $10^{-3}-10^{-4}$. Transfer between non-lysogenic clones of strain 1030 occurred at a similar frequency in urine, and at a frequency of $\sim 10^{-1}$ in serum.

The resistance determinant for transfer between non-lysogenic clones was usually linked to phage genome, as shown by the possession by resistant recipients of immunity to typing phage 75 , plaque-forming particles in their culture filtrates and inducibility by mitomycin C. Stability of the resistance on storage and transduction kinetics suggested that these genes were chromosomal.

Two resistant derivatives were isolated that had lost some phage functions 
and were unable to transfer their resistance further. The epidemiology of gentamicin resistance may in part be explicable by the transient formation of an auto-transmissible element with subsequent integration of the resistance genes into a variety of replicons (i.e., transposition).

I thank Professor D. C. E. Speller and Drs A. J. Bint, A. Howard, R. Warren and D. C. Shanson for providing cultures.

\section{REFERENCES}

Bint, A. J., George, R. H., Healing, D. E., Wise, R. and Davies, M. 1977. An outbreak of infection caused by a gentamicin-resistant Staphylococcus aureus. J. clin. Path., 30, 165.

Buckwold, F. J., Albritton, W. L., Ronald, A. R., Lertzman, J. and Henriksen, R. 1979. Investigations of the occurrence of gentamicin-resistant Staphylococcus aureus. Antimicrob. Agents Chemother., 15, 152.

DowDING, J. E. 1977. Mechanisms of gentamicin resistance in Staphylococcus aureus. Antimicrob. Agents Chemother. 11, 47.

Faden, H., Neter, E., Mclaughlin, S. and Giacoia, G. 1979. Gentamicin-resistant Staphylocaccus aureus. Emergence in an intensive care nursery. J. Am. med. Ass., 241, 143.

Greenhood, G. P., Hill, D., Dixon, R. E., Carter, M. J. and Kanto, W. P. 1979. Changing phage typing patterns of epidemic gentamicin-resistant Staphylococccus aureus. Evidence for transmission of gentamicin resistance. Lancet, 1, 289.

LACEY, R. W. 1971. High-frequency transfer of neomycin resistance between naturally occurring strains of Staphylococcus aureus. J. med. Microbiol., 4, 73.

LACEY, R. W. 1972. Genetic control in methicillin-resistant strains of Staphylococcus aureus. J. med. Microbiol., 5, 497.

LACEY, R. W. 1975. Antibiotic resistance plasmids of Staphylococcus aureus and their clinical importance. Bact. Rev., 39, 1.

LACEY, R. W. 1980. Plasmid transfer in Staphylococcus aureus: evidence for transduction and conjugation. J. gen. Microbiol., in the press.

Lewis, S. A. AND Altemeier, W. A. 1978. Emergence of clinical isolates of Staphylococcus aureus resistant to gentamicin and correlation of resistance with bacteriophage type. $J$. infect. Dis., 137, 314.

Michel, J., Stessman, J. and Sacks, T. 1978. Phenotypic variations in gentamicin-resistant isolates of Staphylococcus aureus. Chemotherapy, 24, 314.

NaIDOO, J. AND NoBLE, W. C. 1978. Transfer of gentamicin resistance between strains of Staphylococcus aureus on skin. J. gen. Microbiol., 107, 391.

Novick, R. 1967. Properties of a cryptic high-frequency transducing phage in Staphylococcus aureus. Virology, 33, 155.

Phillips, S. AND Novick, R. P. 1979. Tn554-a site specific repressor-controlled transposon in Staphylococcus aureus. Nature, London, 278, 476.

Porthouse, A., Brown, D. F. J., SMITH, R. G. AND Rogers, T. 1976. Gentamicin resistance in Staphylococcus aureus. Lancet, 1, 20.

SAXE, M. DE, AND PORTHOUSE, A. 1979. Gentamicin resistance in Staphylococcus aureus. Lancet, 11, 370.

Scott, D. F., Wood, D. O., Brownell, G. H., Carter, M. J. and Best, G. K. 1978. Aminoglycoside modification by gentamicin-resistant isolates of Staphylococcus aureus. Antimicrob. Agents Chemother., 13,641.

Shanson, D. C., KENSIT, J. G. AND DuKE, R. 1976. Outbreak of hospital infection with a strain of Staphylococcus aureus resistant to gentamicin and methicillin. Lancet, 2, 1347.

Soussy, C. J., Dublanchet, A., Cormier, M., Bismuth, R., Mizon, F., Chardon, H., Duval, J. AND FABIANI, G. 1975a. Nouvelles résistances plasmidiques de Staphylococcus aureus aux aminosides. Now. Presse méd., 5, 2599. 
Soussy, C. J., Bouanchaud, D. H., Fouace, J., Dublanchet, A. And Duval, J. 1975b. A gentamicin resistance plasmid in Staphylococcus aureus. Annls Microbiol., Inst. Pasteur: 126B, 91.

Speller, D. C. E., Raghunath, D., Stephens, M., Viant, A. C., Reeves, D. S., Wilkinson, P. J., Broughall, J. M. and Holt, H. A. 1976. Epidemic infection by a gentamicinresistant Staphylococcus aureus in three hospitals. Lancet, 1, 464.

Warren, R. E. AND RoberTs, S. O. B. 1976. Gentamicin-resistant staphylococci. Lancet, 1, 543.

WoOd, D. O., Carter, M. J. And BeSt, G. K. 1977. Plasmid-mediated resitance to gentamicin in Staphylococcus aureus. Antimicrob. Agents Chemother., 12, 513.

Wyatt, T. D., Ferguson, W. P., Wilson, T. S. and McCormick, E. 1977. Gentamicin resistant Staphylococcus aureus associated with the use of topical gentamicin. $J$. antimicrob. Chemother., 3, 213. 